\title{
Estimating Preferences under Risk: The Case of Racetrack Bettors
}

\section{Bruno Jullien}

Institut d'Economie Industrielle, Université de Toulouse, Centre National de la Recherche Scientifique, and Centre for Economic Policy Research

\section{Bernard Salanié}

Centre de Recherche en Economie et Statistique, Centre for Economic Policy Research, and Centre National de la Recherche Scientifique

\begin{abstract}
In this paper we investigate the attitudes toward risk of bettors in British horse races. The model we use allows us to go beyond the expected utility framework and to explore various alternative proposals by estimating a multinomial model on a 34,443-race data set. We find that rank-dependent utility models do not fit the data noticeably better than expected utility models. On the other hand, cumulative prospect theory has higher explanatory power. Our preferred estimates suggest a pattern of local risk aversion similar to that proposed by Friedman and Savage.
\end{abstract}

\section{Introduction}

The attitude toward risk of economic agents has long been the object of considerable attention. The emergence of the so-called nonexpected utility models has challenged the dominant expected utility paradigm and resulted in a large body of theoretical work. This

We are grateful to Hyun Song Shin for helping us to get started on this project and to Tim Drakeford of RACEDATA for providing us with the data we used in this paper. We thank Colin Camerer, Peter Wakker, an anonymous referee, and the editor for their comments. We also thank seminar audiences in Amsterdam, Brussels, Caltech, Chicago, London, Paris, Stanford, and Toulouse. 
literature was primarily motivated by various "paradoxes" emerging from experiments. While the experimental literature on choices under uncertainty is abundant (see, e.g., Hey and Orme 1994; Wu 1994; Camerer 1995; Carbone and Hey 1995; Fennema and Wakker 1996), there are few contributions that compute econometric estimates of non-expected utility models on real-life data sets. The goal of this paper is to fill this gap, using data on the U.K. betting market for horse races.

One main issue concerning empirical work on risk attitudes is that there are few markets for which one can reasonably expect to solve for optimal individual behavior and to collect the relevant information. Financial markets ${ }^{1}$ are intrinsically dynamic, and the extension of non-expected utility models to dynamics is still a controversial issue (see Machina 1989). The insurance market is a very natural candidate and should be exploited in future work (see in particular Cicchetti and Dubin [1994] for a microeconometric estimate of expected utility). ${ }^{2}$ The betting market for horse races presents several advantages for our purpose. In the U.K. market, bookmakers quote odds (prices) that are binding contingent payments for a one-unit bet. This is thus similar to a one-period financial market: betting on one horse is indeed formally analogous to buying a contingent claim. Moreover, the ex post empirical rates of return are observed. The final advantage is that we have access to a very large data set (34,443 races). Many papers have exploited these features and studied horse betting. Some of these papers have focused on testing the rationality of bettors or the efficiency of the market by trying to pinpoint patterns in the data (e.g., Griffith 1949; Dowie 1976; Ali 1979). Some other papers have investigated the extent of insider trading in horse races (e.g., Crafts 1985; Shin 1993; Jullien and Salanié 1994). All these papers draw on the analogy between horse races and financial markets; we elaborate on this analogy by considering horse races as a test bed for alternative theories of behavior under risk. Our goal in this paper is to estimate the parameters of the utility functional of the bettors; it is therefore more closely related to the papers by Weitzman (1965) and Ali (1977), even though they estimated only the parameters of an expected utility functional. There are, however, two drawbacks to our approach. First, we have no data

${ }^{1}$ See in particular the work of Weil (1990), Epstein and Zin (1991), and Jorion and Giovannini (1993) on intertemporal extensions of expected utility models.

${ }^{2}$ There is now an extensive literature investigating the implications of nonexpected utility preferences for the insurance market (see in particular Gollier and Machina [1995]). 
on the characteristics of individual bettors. ${ }^{3}$ Second, prices for combined bets are not available in our data set. For this reason, we follow most of the literature on horse races by focusing on a particular segment of the market, namely on win bets. ${ }^{4}$

Despite these drawbacks, our estimates yield striking and clear results. First, our estimates of the rank-dependent expected utility model (Quiggin 1982) do not significantly differ from those of an expected utility, slightly risk-loving functional (similar conclusions were drawn by Carbone and Hey [1995] and Fennema and Wakker [1996] on experiments). On the other hand, a cumulative prospect theory representation (Kahneman and Tversky 1979; Tversky and Kahneman 1992; Wakker and Tversky 1993) clearly outperforms expected utility. Moreover, the preferences estimated present the pattern of risk attitude that theory and the results from experiments suggest. In particular, they exhibit a form of risk aversion for losses (due to the cumulative weighting), and local utility functions have the shape advocated by Friedman and Savage (1948).

The paper is organized as follows. Section II sets up the general model. Section III presents the data and their main characteristics. Section IV presents the estimation procedure. Section $\mathrm{V}$ gives the estimation results. Section VI then discusses our findings and analyzes risk attitudes. Section VII presents concluding remarks.

\section{The Model}

For any given race, the decision process of bettors can conceivably be decomposed into three steps: (1) The agents decide whether or not to bet. (2) They decide how much to bet. (3) They decide on which horse to bet.

We do not model step 1 in this paper. It presumably involves a utility of gambling that clearly depends on the characteristics of the race, and we have little idea of how to specify it. Regarding step 2, we shall assume that every bettor bets the same amount in all circumstances (we shall denote it $a$ later on). Given that we have no information on the amounts bet, this is a necessary assumption. As for step 3, we assume that bettors do not spread their bets among several horses.

\footnotetext{
${ }^{3}$ We thus follow Weitzman (1965) and Ali (1977) in that we estimate the preferences of a "typical" bettor. We avoid using a full representative agent assumption, however.

${ }^{4}$ Also, optimal combined strategies, even for expected utility maximizers, are complex and extremely difficult to evaluate, and simple bets appear to be the most common practice (see the collection of articles in Hausch, Lo, and Ziemba [1994]).
} 
Some bettors may be noise traders, who bet randomly; others may be insider traders, who have private information. Our paper rests on a representative agent assumption applied to a subgroup of the bettors. We shall assume that there exists a group of identical bettors with initial wealth $M$ who bet $a$ monetary units (say, $£ a$ ) in each race. They are endowed with identical attitudes toward risk, given by a functional $V(F)$, so that if $F_{1}$ and $F_{2}$ are two risky distributions, a bettor will prefer $F_{2}$ to $F_{1}$ if and only if $V\left(F_{2}\right) \geq V\left(F_{1}\right)$. All bettors in this group are endowed with identical beliefs as regards the outcome of the race: they are denoted $\mathbf{p}=\left(p_{1}, \ldots, p_{n}\right)$, where $p_{i}$ is the prior probability that horse $i$ wins the race, and of course $\sum_{i=1}^{n} p_{i}=1$. We assume that these beliefs are unbiased in that the winner is indeed drawn from the $n$ horses according to the probability distribution p. We shall also need to assume later on that every horse, in every race, is bet on by at least one member of the group.

There are many sorts of bets in horse races. In this paper, we shall focus on the simplest form in which bettors try to guess which horse will win the race. Our data pertain to British horse races, in which bets are placed with a bookmaker who offers odds on all horses. Let $R_{i}$ be the rate of return on horse $i .^{5}$ Then betting $£ a$ on horse $i$ brings returns $a R_{i}$ if the horse wins, ${ }^{6}$ which happens with probability $p_{i}$, and $-a$ if another horse wins, which has probability $1-p_{i}$. Therefore, the distribution of returns $F_{i}$ is completely characterized by the pair $\left(p_{i}, R_{i}\right)$, and we can denote the value of such a bet by $W\left(p_{i}, R_{i}\right)=$ $V\left(F_{i}\right)$.

\section{The Data}

Our data are a small part of the data collected by RACEDATA Limited in Britain. For each flat horse race run in Britain between 1986 and 1995, we have the number of horses and the odds (as given by the "starting prices") of all horses in order of arrival.

Our data contain 34,443 races. There is a large variability in the data. The average number of horses in a given race is 10.7. The minimum is two horses (for as many as 133 races) and the maximum is 40 horses (for only one race; there are only 12 races with more than 30 horses). Most races (90 percent of them) have between five and 20 horses.

It is interesting to take a look at the rates of return of our 367,408 horses. Our sample contains both huge favorites (one of them with

\footnotetext{
${ }^{5}$ For instance, a horse quoted at 3 to 2 brings a rate of return of $3 / 2=1.5$ : for every pound bet, if the horse wins, the bettor gets the pound he bet plus $£ 1.5$.

${ }^{6}$ Earnings are not taxed in Britain if the bet is placed at the track.
} 


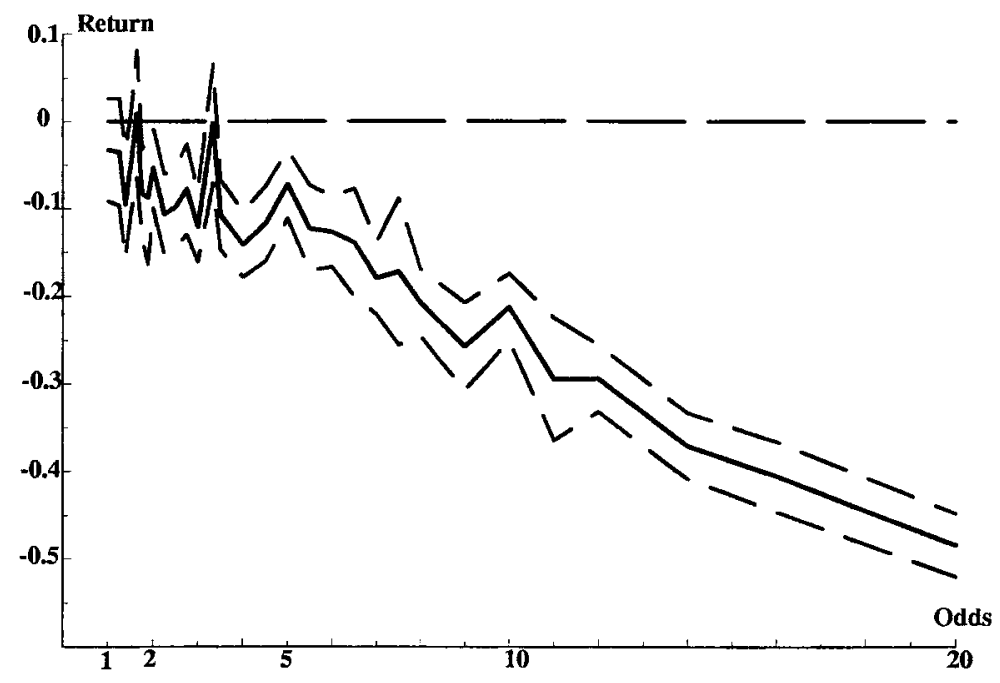

Fig. 1.-Observed expected return

odds of 1 to 50 [in British terms, "50 to 1 on"] - it duly won its race) and very long outsiders (with odds up to 1,000 to 1-none of them won a race); but more than 90 percent of all horses had odds between 1 to 1 and 20 to 1 . Of course, winners tend to have shorter odds. Seventy percent of all big favorites (with odds shorter than 1 to 2) won their race, whereas less than 3 percent of outsiders (with odds longer than 10 to 1 ) won theirs. ${ }^{7}$ However, there are many more outsiders than big favorites, so that 15 percent of all races were won by horses with odds longer than 10 to 1 . The longest outsider to win had odds of 100 to 1 .

Given these data, what is the expected return of betting on a horse with given odds? Figure 1 answers this question. For any given $R$ (we examined each $R$ from 0.02 to 20 by steps of 0.01 ) that corresponds to more than 1,000 horses in our sample, we compute $\hat{p}(R)$, the proportion of horses with rate of return $R$ that won their race. The expected return then is

$$
\overline{\mathrm{ER}}(R)=\hat{p}(R)(R+1)-1 .
$$

Figure 1 plots $\overline{\mathrm{ER}}(R)$, along with a 95 percent confidence interval. It clearly shows that it is not a good idea for a risk-neutral bettor to bet on outsiders (horses with a large $R$ ); this is of course well known, and it is called the favorite/long shot bias in the literature.

${ }^{7}$ As expected, the proportion of winners in each odds class decreases regularly with the odds. 


\section{The Estimation Procedure}

Our goal is to recover information on the parameters of the attitudes toward risk of our select group of bettors. We therefore introduce a parameter vector $\boldsymbol{\theta}$ that describes the preferences of the bettors, and we now denote them $V(F, \boldsymbol{\theta})$ and the value of betting $£ a$ on horse $i$ by $W\left(p_{i}, R_{i}, \boldsymbol{\theta}\right)$ (suppressing the dependence on $a$ for simplicity).

Our estimation procedure first involves solving for the probabilities $p_{i}$ as functions of the rates of return $R_{i}$ and the parameter vector $\boldsymbol{\theta}$. Then we set up and evaluate a multinomial model based on which horse won the race.

\section{A. Solving the Model}

Since all bettors in our group are identical, the odds must be such that they leave them indifferent between all horses. Otherwise, there would be two horses, say $i$ and $j$, for which

$$
W\left(p_{i}, R_{i}, \boldsymbol{\theta}\right)>W\left(p_{j}, R_{j}, \boldsymbol{\theta}\right),
$$

and none of the bettors would bet on horse $j$ since we assume that bettors do not spread their bets. Since we assume that all horses are bet on by at least one member of the group at equilibrium, in every race, $W\left(p_{i}, R_{i}, \boldsymbol{\theta}\right)$ must be independent of $i$ :

$$
W\left(p_{1}, R_{1}, \boldsymbol{\theta}\right)=\cdots=W\left(p_{n}, R_{n}, \boldsymbol{\theta}\right) .
$$

This set of equalities is the basis of our estimating procedure. ${ }^{8}$ We prove in the Appendix that given two mild restrictions on the functional $V$ (continuity and first-order stochastic dominance), this set of equalities defines each $p_{i}$ uniquely as a function of all the $R_{i}$ 's and $\boldsymbol{\theta}$.

This shows that it is possible to recover the probabilities of a win by each horse from the observation of the odds on each horse and the condition of market equilibrium. Note that we imposed very little on the bettors' attitude toward risk: most of the functional forms for $V$ that have been proposed in the literature indeed exhibit firstorder stochastic dominance and continuity.

\footnotetext{
${ }^{8}$ Note that it also holds if there are insider traders (who have different priors) or noise traders (who bet randomly), provided only that the group of identical bettors spread their bets over all horses.
} 


\section{B. Examples}

We now show how the $p$ functions can be obtained in three popular classes of models that we shall estimate later.

\section{Example 1: Expected Utility}

Assume that the functional $V(F)$ takes the familiar expected utility form:

$$
V(F, \boldsymbol{\theta})=\int u(x, \boldsymbol{\theta}) d F(x) .
$$

Then the value of betting on horse $i$ for a bettor with initial wealth $M$ is

$$
W\left(p_{i}, R_{i}, \boldsymbol{\theta}\right)=p_{i} u\left(M+a R_{i}, \boldsymbol{\theta}\right)+\left(1-p_{i}\right) u(M-a, \boldsymbol{\theta}),
$$

and solving the equation $W\left(p_{i}, R_{i}, \boldsymbol{\theta}\right)=w$ gives

$$
p_{i}=\frac{w-u(M-a, \boldsymbol{\theta})}{u\left(M+a R_{i}, \boldsymbol{\theta}\right)-u(M-a, \boldsymbol{\theta})} .
$$

Finally, the adding-up constraint $\sum_{i=1}^{n} p_{i}=1$ gives

$$
w=u(M-a, \boldsymbol{\theta})+\frac{1}{\sum_{i=1}^{n} \frac{1}{u\left(M+a R_{i}, \boldsymbol{\theta}\right)-u(M-a, \boldsymbol{\theta})}},
$$

and we obtain an explicit formula for the probabilities:

$$
p_{i}=\frac{1}{u\left(M+a R_{i}, \boldsymbol{\theta}\right)-u(M-a, \boldsymbol{\theta})} \frac{1}{\sum_{j=1}^{n} \frac{1}{u\left(M+a R_{j}, \boldsymbol{\theta}\right)-u(M-a, \boldsymbol{\theta})}} .
$$

Example 2: Anticipated Utility

As a first generalization of expected utility, consider the following formulation, which Quiggin (1982) calls anticipated utility (for a special case) and which other authors call rank-dependent expected utility (RDEU):

$$
V(F, \boldsymbol{\theta})=-\int u(x, \boldsymbol{\theta}) d[G \circ(1-F)](x),
$$

where $G$ is a continuous increasing function that maps $[0,1]$ into itself. 
The value of a bet on horse $i$ then is

$$
\begin{aligned}
W\left(p_{i}, R_{i}, \boldsymbol{\theta}\right)= & G\left(p_{i}, \boldsymbol{\theta}\right) u\left(M+a R_{i}, \boldsymbol{\theta}\right) \\
& +\left[1-G\left(p_{i}, \boldsymbol{\theta}\right)\right] u(M-a, \boldsymbol{\theta}) .
\end{aligned}
$$

Writing the equation $W\left(p_{i}, R_{i}, \boldsymbol{\theta}\right)=w$, we get

$$
G\left(p_{i}, \boldsymbol{\theta}\right)=\frac{w-u(M-a, \boldsymbol{\theta})}{u\left(M+a R_{i}, \boldsymbol{\theta}\right)-u(M-a, \boldsymbol{\theta})} .
$$

Let $H(\cdot, \boldsymbol{\theta})$ be the inverse function of $G(\cdot, \boldsymbol{\theta})$ (which exists since the latter is continuous and increasing); the adding-up constraint $\sum_{i=1}^{n} p_{i}=1$ gives

$$
\sum_{i=1}^{n} H\left(\frac{w-u(M-a, \boldsymbol{\theta})}{u\left(M+a R_{i}, \boldsymbol{\theta}\right)-u(M-a, \boldsymbol{\theta})}, \boldsymbol{\theta}\right)=1 .
$$

Solving this equation gives $w$ and therefore the $p_{i}$ 's.

\section{Example 3: Cumulative Prospect Theory}

Prospect theory was introduced by Kahneman and Tversky (1979) and developed into cumulative prospect theory (CPT) in Tversky and Kahneman (1992). This theory differs from other theories in that it evaluates changes in wealth with respect to a reference point. ${ }^{9}$ The initial wealth $M$ here is a natural reference point. Let $u$ be a continuous increasing function such that $u(0)=0$ and $G$ and $H$ be two continuous increasing functions that map $[0,1]$ into itself. Then the value of betting on horse $i$ is

$$
W\left(p_{i}, R_{i}, \boldsymbol{\theta}\right)=G\left(p_{i}, \boldsymbol{\theta}\right) u\left(a R_{i}, \boldsymbol{\theta}\right)+H\left(1-p_{i}, \boldsymbol{\theta}\right) u(-a, \boldsymbol{\theta}) .
$$

Clearly, rank-dependent utility is the special case in which $G(p)+$ $H(1-p)=1$. Another interesting case is that of "reflection," in which $G=H$.

Solving for the $p_{i}$ 's here is slightly more complicated than in example 2 since even solving for $p$ as a function of $w$ can usually not be done in a closed form. However, the procedure remains the same.

Note that all these examples share the property that the function $P\left(w, R_{i}, \boldsymbol{\theta}\right)$ can be obtained explicitly or with a simple numerical procedure, so that there remains only to find the solution $w$ to $\sum_{i} P\left(w, R_{i}, \boldsymbol{\theta}\right)=1$, which is a fairly simple numerical task since the left-hand side is increasing and we know that $w$ must lie in the interval $\left(W\left(0, R_{i}, \boldsymbol{\theta}\right), W\left(1, \min _{j} R_{j}, \boldsymbol{\theta}\right)\right)$.

\footnotetext{
${ }^{9}$ Early forms of prospect theory could violate first-order stochastic dominance, but CPT does not.
} 


\section{Estimating the Parameters}

Since we know which horse won the race (it is always $i=1$ in our notation) and given that we can compute the $p_{i}$ 's, it is a simple matter to set up a multinomial model. Let $c=1, \ldots, C$ be an index that runs over all races in our sample. Then the log likelihood for the sample is simply

$$
L^{C}(\boldsymbol{\theta})=\sum_{c=1}^{C} \log p_{1}\left(R^{c}, \boldsymbol{\theta}\right) .
$$

The usual properties apply, so that maximizing $L^{C}$ gives us an estimator $\hat{\boldsymbol{\theta}}^{C}$ that is consistent, asymptotically normal, and asymptotically efficient:

$$
\sqrt{C}\left(\hat{\boldsymbol{\theta}}^{C}-\boldsymbol{\theta}_{0}\right) \rightarrow N\left(0, I^{-1}\right),
$$

where $I$ can be consistently estimated by

$$
\hat{I}=\frac{1}{C} \sum_{c=1}^{C} \frac{1}{\left[p_{1}\left(R^{c}, \hat{\boldsymbol{\theta}}^{C}\right)\right]^{2}} \frac{\partial p_{1}}{\partial \boldsymbol{\theta}}\left(R^{c}, \hat{\boldsymbol{\theta}}^{C}\right) \frac{\partial p_{1}}{\partial \boldsymbol{\theta}^{\prime}}\left(R^{c}, \hat{\boldsymbol{\theta}}^{C}\right) .
$$

\section{The Results}

Here we apply the preceding model and estimation procedure to the data described in Section III.

\section{A. Expected Utility Models}

We tested our procedure on the most common von Neumann-Morgenstern utility functions. This will also allow us to explain the identification problems that arise because of our not knowing the initial wealth $M$ and the size of the bet $a$.

Recall the formula for the probabilities:

$$
\begin{aligned}
p_{i}= & \frac{1}{u\left(M+a R_{i}, \boldsymbol{\theta}\right)-u(M, a, \boldsymbol{\theta})} \\
& \times \frac{1}{\sum_{j=1}^{n} \frac{1}{u\left(M+a R_{j}, \boldsymbol{\theta}\right)-u(M-a, \boldsymbol{\theta})}} .
\end{aligned}
$$

First consider the constant absolute risk aversion (CARA) utility function $u(x, \theta)=\left(1-e^{-\theta x}\right) / \theta$. Obviously, the factors $e^{-\theta M}$ cancel out in the expression for $p_{i}$, and it is easy to see that $p_{i}$ depends only on the product $\theta a$. Thus $\theta$ is not identifiable in this model; only $\theta a$ 
is. Maximizing $L^{C}$ gives $\hat{\theta}^{C} a=-0.055$, with standard error 0.001 . The value of $L^{C}$ at the optimum is $-63,801.2$. Thus bettors appear to be risk-loving. ${ }^{10}$ However, while $\hat{\theta}^{C}$ is significantly negative, it has a very small absolute value: even if the size of the representative bet is only $£ 1$ (which is probably an underestimate), a value of $-\hat{\theta}^{C}$ close to 0.06 does not seem to imply very bold risk taking. Standard ArrowPratt calculations indeed yield, for a bet whose standard error is $a$ (the unknown amount bet in horse races), a (negative) risk premium $\rho$ given by $\rho / a=\theta a / 2$ so that the risk premium is only about 2.8 percent of $a$.

A more general specification is the hyperbolic absolute risk aversion (HARA) family, given by

$$
u(x, \theta)=\left(\theta_{1}+x\right)^{\theta_{2}}
$$

which yields an absolute risk aversion index

$$
-\frac{u^{\prime \prime}}{u^{\prime}}=\frac{1-\theta_{2}}{\theta_{1}+x}
$$

and thus nests, inter alia, both the CARA and constant relative risk aversion (CRRA) families.

Simple computations show that $p_{i}$ depends only on $\theta_{2}$ and $\left(\theta_{1}+\right.$ $M) / a$. Maximizing $L^{C}$ gives strange estimates: both $\left(\hat{\theta}_{1}^{C}+M\right) / a$ and $\hat{\theta}_{2}^{C}$ appear to be very large and have large standard errors. A closer look at the shape of $L^{C}$ shows that it is maximal and almost constant on a curve on which $\theta_{2} a /\left(\theta_{1}+M\right)$ is constant and close to 0.06 . But for $\left(\theta_{1}+M\right) / a$ and $\theta_{2}$ large on that curve,

$$
\left(1+\frac{a}{\theta_{1}+M} x\right)^{\theta_{2}} \simeq \exp \left(\frac{a \theta_{2}}{\theta_{1}+M} x\right) \simeq \exp (0.06 x),
$$

so that we are back to our estimate for the CARA family. ${ }^{11}$ The value of $L^{C}$ at the optimum is $-63,796.5$, which is significantly larger than that obtained for the optimal CARA utility function; however, the difference is not huge given that we have such a large number of observations. Moreover, the qualitative behavior implied by these estimates does not differ very much from the CARA case. ${ }^{12}$ We therefore conclude that the CARA utility is close to providing the best fit

\footnotetext{
${ }^{10}$ This was not a foregone conclusion since we do not model the decision to bet.

${ }^{11}$ This argument is corroborated by the fact that the estimated correlation between $\hat{\theta}_{1}^{C}$ and $\hat{\theta}_{2}^{C}$ is very close to one.

${ }^{12}$ It is easy to see that the implied absolute risk aversion index, multiplied by $a$ and evaluated in $x=M$, is -0.063 .
} 
TABLE 1

Rank-Dependent Utility: Power Function

\begin{tabular}{lcc}
\hline \hline Parameter & Estimate & Standard Error \\
\hline$\theta a$ & -.053 & .003 \\
$\alpha$ & .988 & .016 \\
\hline
\end{tabular}

among all HARA utility functions, better than, say, a CRRA function. $^{13}$

Given these preliminary results and since we are mainly interested in non-expected utility theories, whose main focus is on how agents "weigh" probabilities, we shall take the value function to be CARA from now on:

$$
u(x, \theta)=\frac{1-\exp (-\theta x)}{\theta},
$$

which is zero at $x=0$. This actually simplifies our task since it means that initial wealth $M$ divides out in the formulas for probabilities, and thus we do not have to worry about the fact that we do not observe it.

\section{B. Rank-Dependent Utility}

Let us now focus on our example 2, in which agents weigh probabilities in a systematic way given by the function $G$. Given that our estimation process is fairly costly (the estimates above took about two CPU hours on a UNIX minicomputer), we shall choose specifications for $G$ that are easily invertible.

Our first candidate is just the power function $G(p)=p^{\alpha}$. We obtain the estimates in table 1.

The value of the likelihood is $-63,801.0$, which is not significantly higher than the expected utility CARA specification. The power coefficient $\hat{\alpha}$ is very close to and not significantly different from one, and the CARA estimate is hardly modified..$^{14}$

Of course, the power function allows for only a wholly concave or wholly convex function $G$, whereas the literature points toward a function that is concave then convex (so that it overweighs small

\footnotetext{
${ }^{13}$ Thus our results cannot be directly compared to those of Ali (1977), who estimates only a CRRA specification on his American data set, using a totally different and somewhat contrived procedure. Ali finds an index of relative risk aversion of -0.178 , which does corroborate our finding that bettors are only weakly risk-loving.

${ }^{14}$ Incidentally, the fact that it still significantly differs from zero implies that we reject the dual theory of Yaari (1987).
} 
probabilities and underweighs high probabilities). To describe this, we shall use a specification due to Cicchetti and Dubin (1994):

$$
\frac{G(p)}{1-G(p)}=\left(\frac{p}{1-p}\right)^{a_{1}}\left(\frac{p_{0}}{1-p_{0}}\right)^{1-a_{1}} \text {. }
$$

This increases from zero to one and crosses the diagonal in $p_{0}$; it may be concave then convex (for $a_{1}<1$ ) or convex then concave (for $a_{1}>1$ ). It collapses to the expected utility specification for $a_{1}$ $=1$ (in which case $p_{0}$ is not identified).

Camerer and Ho (1994) use a different specification, in which

$$
G(p)=\frac{p^{\gamma}}{\left[p^{\gamma}+(1-p)^{\gamma}\right]^{1 / \gamma}} .
$$

This allows for changes in concavity but is less flexible than the Cicchetti-Dubin specification since it uses the single parameter $\gamma$ to account for both the curvature of $G$ and the point at which it crosses the diagonal. Also, this $G$ cannot be inverted analytically, which makes estimation very cumbersome. On the other hand, we did estimate the Lattimore-Baker-Witte (1992) specification:

$$
G(p)=\frac{\delta p^{\gamma}}{\delta p^{\gamma}+(1-p)^{\gamma}},
$$

which has two parameters, can be inverted easily, and nests expected utility as $\delta=\gamma=1$.

Finally, we also estimated the specification suggested by Prelec (1998):

$$
G(p)=\exp \left[-\beta(-\ln p)^{\alpha}\right],
$$

which he obtained after imposing several axioms on choice under risk. This nests the power specification (for $\alpha=1$ ) and therefore also expected utility (for $\alpha=\beta=1$ ).

The maximization program took about $20 \mathrm{CPU}$ hours for each specification. The results appear in table 2.

Again, the surprising result is that rank-dependent utility does not improve on expected utility. For the Cicchetti-Dubin specification, the crucial parameter $a_{1}$ does not significantly differ from one, even though it is estimated precisely. As expected, the likelihood function hardly depends on $p_{0}$, and thus its estimated standard error is very large ${ }^{15}$ Its maximal value is $-63,801.1$, which is even slightly worse

\footnotetext{
${ }^{15}$ Remember that $p_{0}$ is the point at which the weighting function crosses the diagonal, and that is not identified for expected utility since the weighting function everywhere coincides with the diagonal.
} 
TABLE 2

Rank-Dependent Utility: Cicchetti/Dubin AND LATTIMORE ET AL. FUnCTIONS

\begin{tabular}{|c|c|c|}
\hline Parameter & Estimate & Standard Error \\
\hline & \multicolumn{2}{|c|}{ Cicchetti-Dubin } \\
\hline$\theta a$ & -.054 & .005 \\
\hline$\alpha_{1}$ & .994 & .032 \\
\hline \multirow[t]{2}{*}{$p_{0}$} & .500 & 5.459 \\
\hline & \multicolumn{2}{|c|}{ Lattimore et al. } \\
\hline$\theta a$ & -.050 & .005 \\
\hline$\gamma$ & .965 & .032 \\
\hline$\delta$ & .877 & .132 \\
\hline
\end{tabular}

TABLE 3

Rank-Dependent Utility: Prelec Function

\begin{tabular}{lcc}
\hline \hline Parameter & Estimate & Standard Error \\
\hline$\theta a$ & -.036 & .007 \\
$\alpha$ & .879 & .051 \\
$\beta$ & 1.143 & .081 \\
\hline
\end{tabular}

than for the less flexible power function. ${ }^{16}$ These results are consistent with those of Cicchetti and Dubin (1994) in the context of insurance and with the experiments of Carbone and Hey (1995) and Fennema and Wakker (1996). For the Lattimore et al. specification, we again find that the estimates are very close to the CARA expected utility specification, and we cannot reject expected utility.

As shown in table 3, the estimates for the Prelec specification tell a slightly different story. The estimated $\alpha$ significantly differs from one, so that the Prelec specification fits the data better than the power specification. The value of the Wald test statistic for the expected utility hypothesis is 12.3 (for two degrees of freedom), so that this time expected utility is rejected in favor of the richer Prelec specification. On the other hand, the estimated weighting function is still rather close to the diagonal, as shown in figure 2. We therefore conclude that while the deviation from expected utility is statistically significant, it is not clear that it is significant in economic terms.

\footnotetext{
${ }^{16}$ In order to check that this is not a numerical failure, we held $\theta a$ and $p_{0}$ constant and plotted the likelihood function. This confirms that the maximum in $a_{1}$ is very close to one.
} 


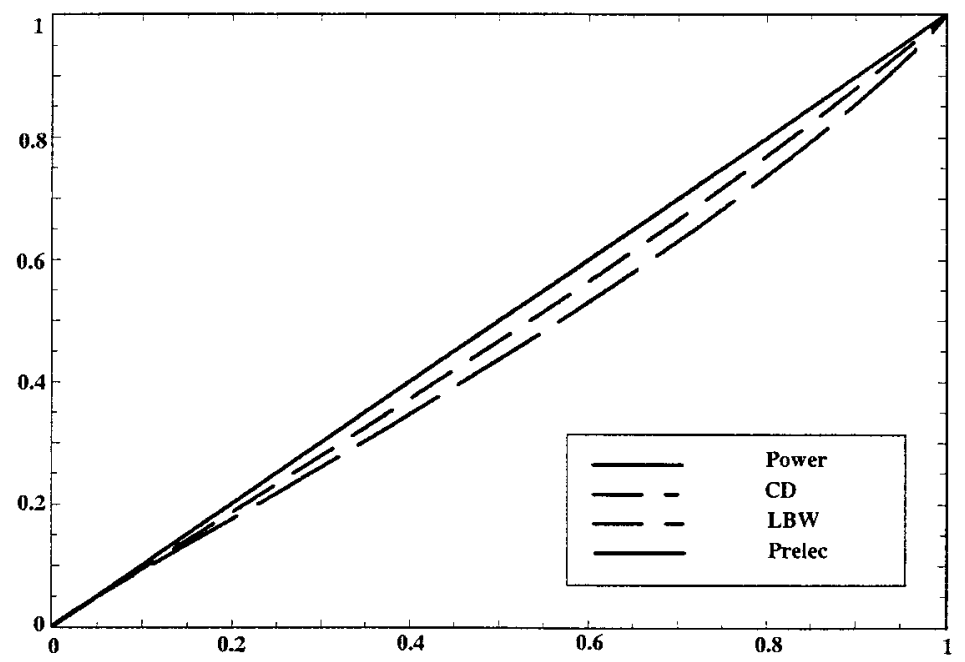

FIG. 2.-Estimated weighting functions (RDEU)

\section{Cumulative Prospect Theory}

We now move to our example 3. This has added generality (as compared to rank-dependent utility) in that agents weigh probabilities in different ways for losses and for gains. ${ }^{17}$ Unfortunately, this also means that even if we choose easily invertible $G$ and $H$ functions, we shall have to solve numerically in the first stage for the $p_{i}$ 's as functions of $w$. This makes the program much more computer-intensive. Indeed, we had to optimize the program in several ways in order to achieve reasonable estimation times: (1) we wrote a root-finding program that starts with a Newton-Raphson algorithm with analytical derivatives and switches to a bisection routine when it wanders out of the theoretical bounds for the root; (2) we computed the gradient of the likelihood function analytically.

Even so, one computation of the likelihood function still takes several CPU hours, given the very large number of multidimensional root-finding calculations involved. Thus we focus on the races that

\footnotetext{
${ }^{17}$ In the context of CPT, it would be natural to allow for a different value function $u$ for gains and for losses. There is indeed evidence (see Camerer 1995) that agents behave as though they were risk-loving for gains and risk-averse for losses. However, we observe only one level of losses $(-a)$, and $a$ is not identifiable, so that there is little we can do in that direction. Therefore, we shall stick to the assumption of a single CARA function for both losses and gains. However, we did replace $u(-1)$ with $u(-1)+\gamma$ and test $\gamma=0$. The resulting score (Lagrange multiplier) statistic is only 0.49 , and thus we cannot reject the hypothesis that the value function for losses is generated from the same CARA function as for gains.
} 
TABLE 4

Cumulative Prospect Theory: Power Functions

\begin{tabular}{lcc}
\hline \hline Parameter & Estimate & Standard Error \\
\hline$\theta a$ & -.072 & .021 \\
$\alpha$ & 1.162 & .143 \\
$\beta$ & .318 & .272 \\
\hline
\end{tabular}

were run in 1995, the last year of our sample. There are 4,037 of them, or about 11.7 percent of the total sample. This reduces the computation time to about 30 minutes per function evaluation, which is still a lot.

We first estimate a specification in which both $G$ and $H$ are power functions: $G(p)=p^{\alpha}$ and $H(p)=p^{\beta}$. The estimation results appear in table 4.

The CARA estimate stays in the same ballpark. The power function for gains is slightly convex, but not significantly so. On the other hand, the power function for losses is highly and significantly concave. This is the first notable deviation from expected utility that we encounter. This is confirmed by a Wald test of the expected utility hypothesis (which is given by $\alpha=\beta=1$ ): the test statistic is 15.04 for two degrees of freedom, which points to a very clear rejection of expected utility.

It is not possible to test for rank-dependent utility in the CPT model with power functions; but we already know that rank-dependent utility does not fit the data better than expected utility, and that is rejected by the data. On the other hand, we can test the reflection hypothesis $\alpha=\beta$; this is also rejected, with a $\chi^{2}(1)$ of 4.31 .

We have also estimated a model in which both $G$ and $H$ take the Cicchetti-Dubin form. Thus

$$
\frac{G(p)}{1-G(p)}=\left(\frac{p}{1-p}\right)^{a_{1}}\left(\frac{p_{0}}{1-p_{0}}\right)^{1-a_{1}}
$$

and

$$
\frac{H(p)}{1-H(p)}=\left(\frac{p}{1-p}\right)^{a_{1}^{\prime}}\left(\frac{p_{0}^{\prime}}{1-p_{0}^{\prime}}\right)^{1-a_{1}^{\prime}} .
$$

The results (obtained after more than one month of maximization) are given in table 5 . Unfortunately, the standard errors are very large so that it is difficult to reach definite conclusions. In particular, it is impossible to reject rank-dependent utility $\left(a_{1}=a_{1}^{\prime}\right.$ and $p_{0}+p_{0}^{\prime}$ $=1)$, the reflection hypothesis $\left(a_{1}=a_{1}^{\prime}\right.$ and $\left.p_{0}=p_{0}^{\prime}\right)$, or even ex- 
TABLE 5

Cumulative Prospect Theory: Cicchetti-Dubin FunCTIONS

\begin{tabular}{lcc}
\hline \hline Parameter & Estimate & Standard Error \\
\hline$\theta a$ & -.065 & .035 \\
$a_{1}$ & 1.087 & .372 \\
$p_{0}$ & .959 & 1.241 \\
$a_{1}^{\prime}$ & .728 & 1.943 \\
$p_{0}^{\prime}$ & .996 & .221 \\
\hline
\end{tabular}

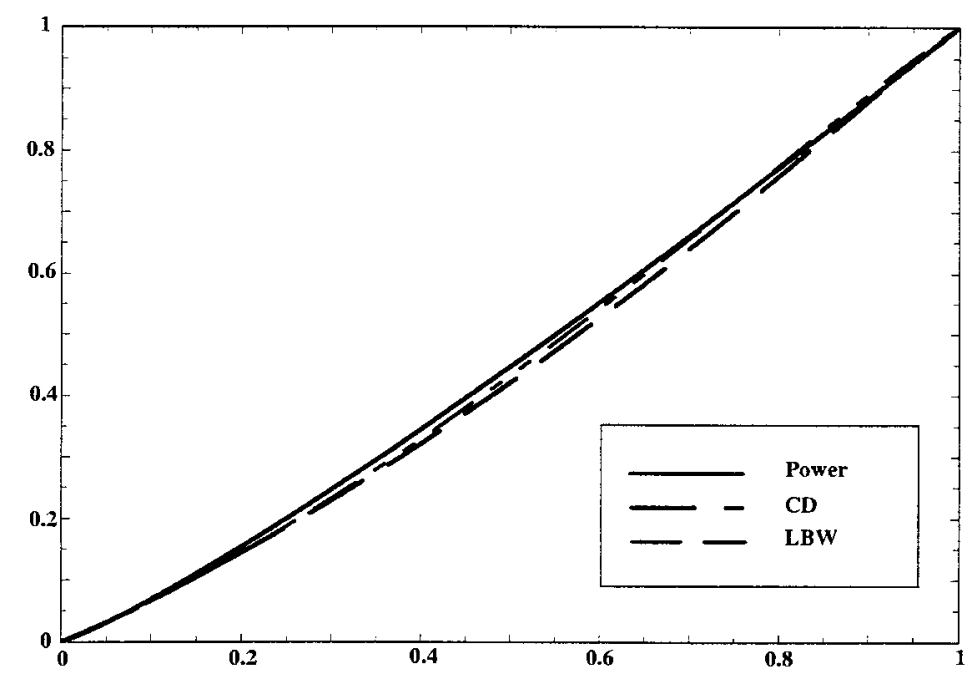

FIG. 3.-Weighting function for gains

pected utility $\left(a_{1}=a_{1}^{\prime}=1\right)$. It seems that the data do not support changing concavity in the weighting functions.

Still, the message conveyed by the point estimates is very similar to that of the model with power functions. ${ }^{18}$ Using the Lattimore et al. specification for both weighting functions confirms these findings. As in the Cicchetti-Dubin case, standard errors are fairly large $;^{19}$ but the estimated $G$ and $H$ weighting functions are very close to those for the power and Cicchetti-Dubin specifications. ${ }^{20}$ Figures 3

\footnotetext{
${ }^{18}$ Also, the value of the likelihood function is not significantly higher, in spite of the two added parameters: $-7,365.25$, as compared to $-7,365.31$.

${ }^{19}$ In both cases, this may be due to our attempt to estimate a change in concavity that is not in the data.

${ }^{20}$ We also tried to use the Prelec specification, but we met with severe numerical difficulties and could not get the algorithm to converge.
} 


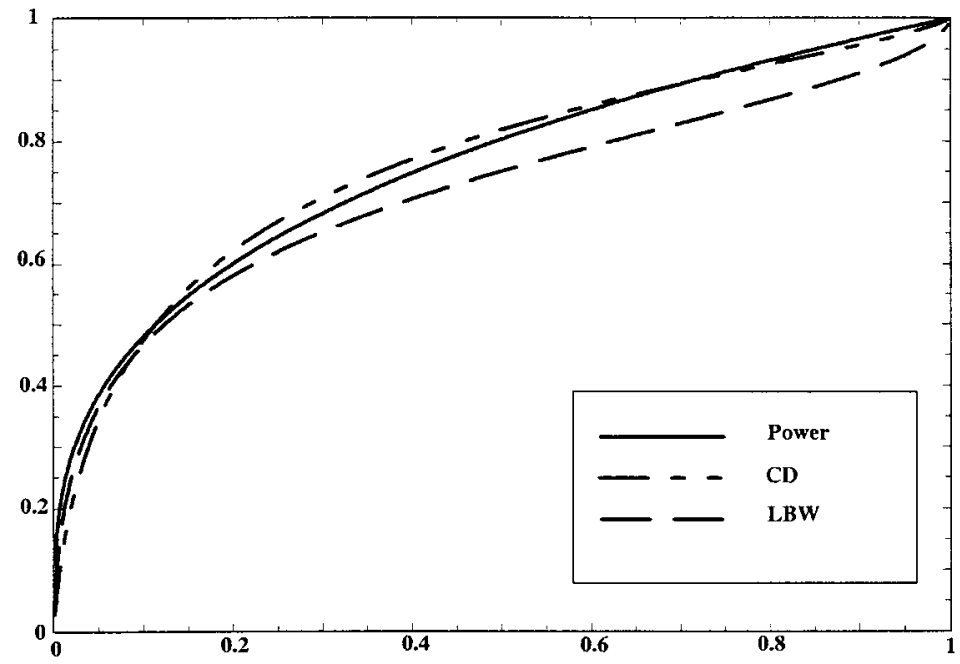

FIG. 4.-Weighting function for losses

and 4 show the estimated $G$ and $H$ functions for the CPT specifications. This shows that contrary to the RDEU case, deviations from expected utility this time are economically significant. It is striking that in all three cases, the $G$ function for gains is slightly convex but close to the bisectrix and the $H$ function for losses is concave on most of the range. Note in particular that even though the CicchettiDubin and Lattimore et al. specifications allow the $G$ and $H$ functions to change concavity, the data do not appear to support this. This is a somewhat surprising result in view of the many experiments that find evidence of changing concavity; in particular, we find little evidence for a "certainty effect'" (convex weighting functions near $p=1)$.

\section{Interpretation}

We shall now explore the economic consequences of our estimation results. To do this, we focus on the CPT model with power functions, for which the estimation results are given in table 4 . This is our preferred model on grounds of fit and parsimony.

\section{A. Fanning Out and the Allais Paradox}

Much of what follows will use the "probability triangle," an expository device developed by Marschak (1950) and later Machina (1987). Consider the set of lotteries with a fixed three-point support 


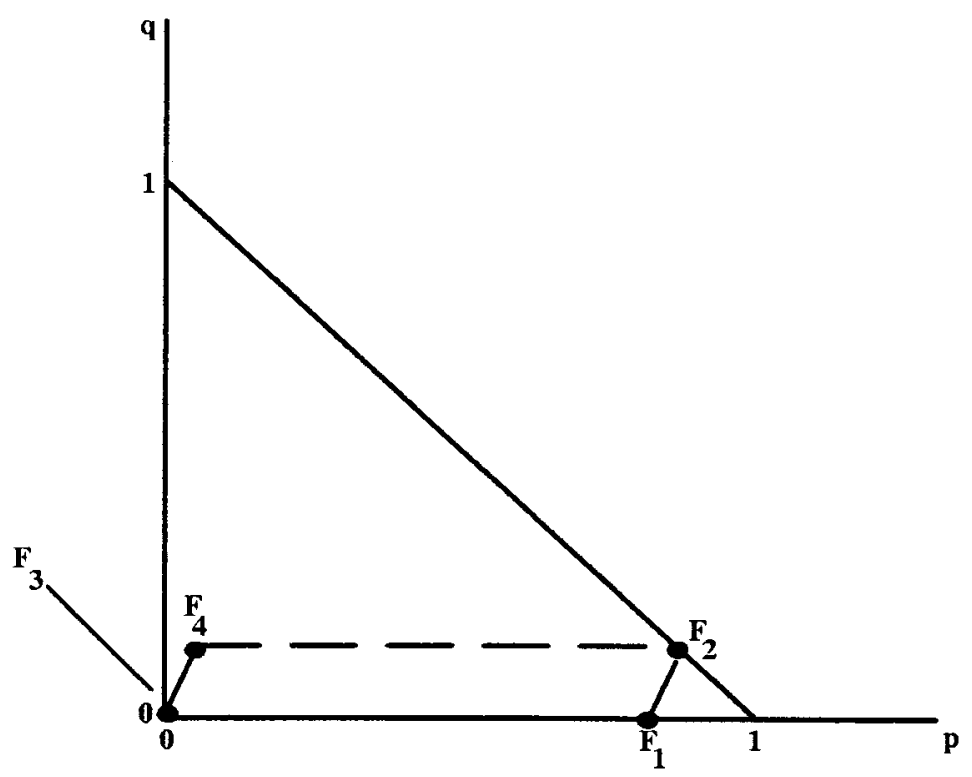

FIG. 5.-Original Allais paradox

$\left\{x_{1}, x_{2}, x_{3}\right\}, x_{1}<x_{2}<x_{3}$. A lottery gives $x_{1}$ with probability $p, x_{2}$ with probability $1-p-q$, and $x_{3}$ with probability $q$. Such a lottery can be represented by a pair $(p, q)$ in the triangle $p+q \leq 1$. In the triangle, preferences can be represented by the indifference curves on lotteries $(p, q)$. Under expected utility, preferences are linear in probabilities, and thus these indifference curves are parallel straight lines. Now consider the four following lotteries, for $\left(x_{1}, x_{2}, x_{3}\right)=$ $(0, \$ 1 \mathrm{M}, \$ 5 \mathrm{M}): F_{1}$ : win $\$ 1 \mathrm{M}$ with probability .11; $F_{2}$ : win $\$ 5 \mathrm{M}$ with probability $.1 ; F_{3}$ : win $\$ 1 \mathrm{M}$ for sure; $F_{4}$ : win $\$ 5 \mathrm{M}$ with probability .1 or $\$ 1 \mathrm{M}$ with probability .89 .

As can be seen in figure $5,{ }^{21} F_{1} F_{2}$ and $F_{3} F_{4}$ define parallel segments, so that according to expected utility, $F_{1}$ should be preferred to $F_{2}$ if and only if $F_{3}$ is preferred to $F_{4}$. However, Allais (1953) showed that many individuals prefer $F_{2}$ to $F_{1}$ and yet prefer $F_{3}$ to $F_{4}$.

The development of non-expected utility models has been strongly influenced by similar experimental evidence on violations of the expected utility hypotheses (see Machina 1987). On the basis of this evidence, Segal (1987) defines what he calls the "generalized Allais paradox" (GAP). The GAP states that if $F_{i}, i=1, \ldots, 4$, denote four lotteries in the triangle such that $F_{1}$ and $F_{3}$ lie on the same hori-

${ }^{21}$ The offsets have been somewhat exaggerated to make them more apparent. 


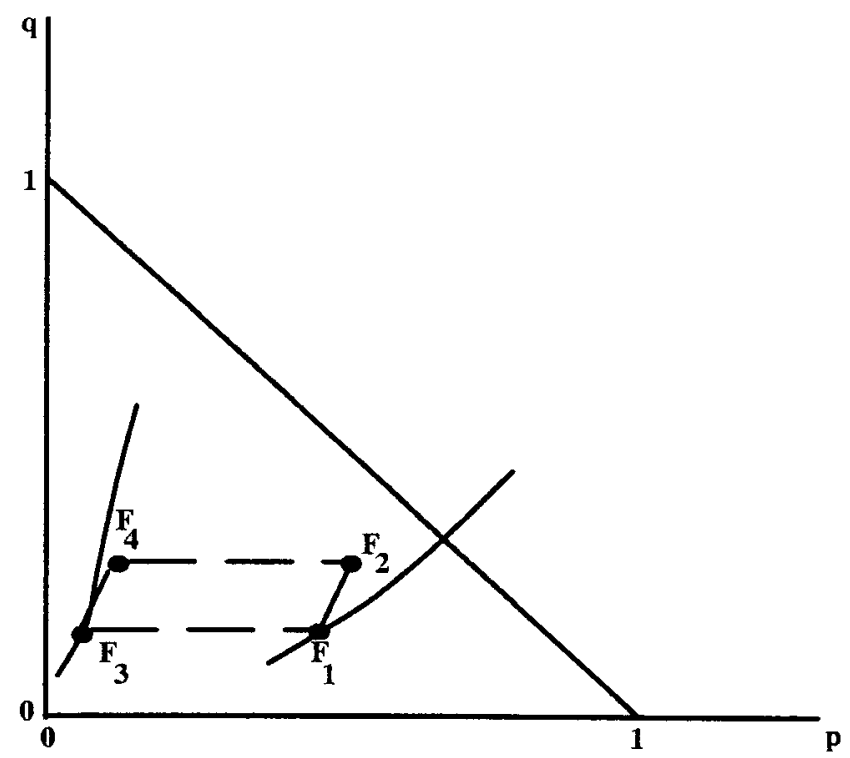

Fig. 6.-Generalized Allais paradox

zontal line and $F_{2}$ and $F_{4}$ lie on the same horizontal line, $F_{4}-F_{3}=$ $F_{2}-F_{1}$, then $V\left(F_{1}\right)=V\left(F_{2}\right)$ implies $V\left(F_{3}\right)>V\left(F_{4}\right)$. Figure 6 illustrates a GAP.

Green and Jullien (1988, p. 369) give conditions for a preference functional to generate the GAP. Specializing their results to CPT shows that in our notation, their condition reduces to requiring that $G$ be convex and $H$ concave, which is indeed true for our estimates. Thus our estimated CPT functional generates behavior that is consistent with the GAP.

Machina (1987) showed that many violations of expected utility theory (Allais paradox, common consequence effect, and common ratio effect) can be explained by assuming that indifference curves fan out in the probability triangle (see fig. 6). Roughly speaking, this means that the slope of the indifference curve should increase as $p$ increases or $q$ decreases. However, more recent experimental research has found that indifference curves exhibit a mixture of fanning out and fanning in. In any case, it is interesting to look at the implied shape of indifference curves in the triangle diagram for our estimates.

To do this, we fix $x_{1}=-1, x_{2}=0$, and $x_{3}=1$. The value of this lottery is

$$
w(p, q)=G(q) u(1)+H(p) u(-1)
$$




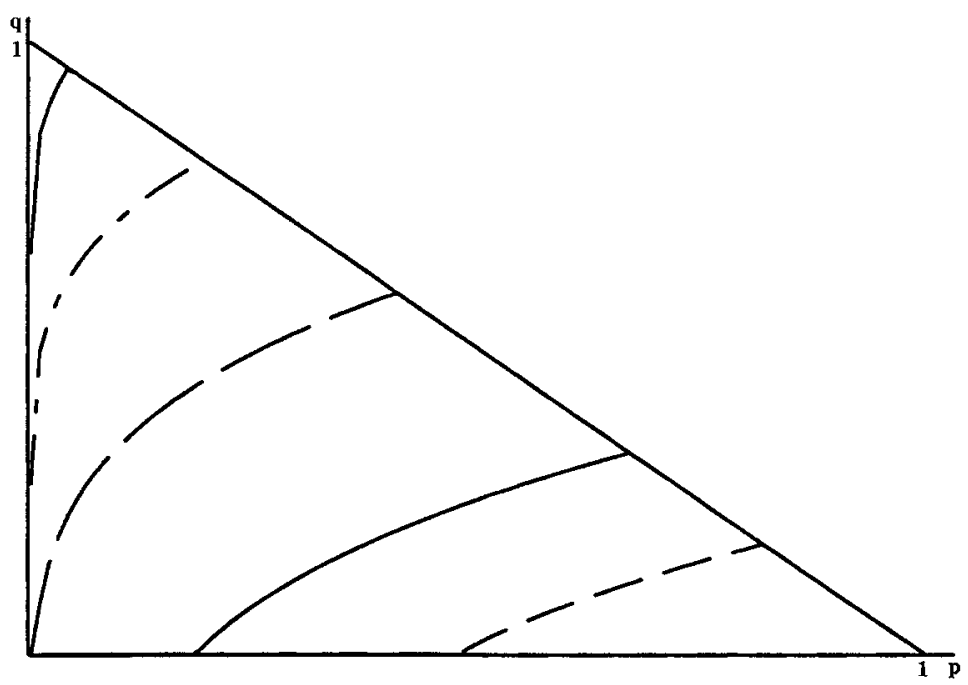

FIG. 7.-Estimated indifference curves

since by construction $u(0)=0$. Figure 7 plots the indifference curves $w(p, q)=k$ in the $(p, q)$ plane.

If fanning out is interpreted in the weak sense that indifference curves get steeper when one moves from the southeast corner to the northwest corner, then clearly our estimates exhibit fanning out. On the other hand, strict fanning out (i.e., the property that, for given $p$, the slope of the indifference curve increases with $k$ ) is rejected: it is easy to prove that, in fact, $\alpha>1$ implies that there is a moderate degree of fanning in this stricter sense. ${ }^{22}$ Moreover, the fanning out property seems to be concentrated at the left edge of the triangle, which again is consistent with experimental evidence, as collected, for instance, in Camerer (1995).

\section{B. Local Utility Functions}

A second key element to analyze the properties of nonlinear preferences is the set of "local utility functions." As defined by Machina (1982), they are the local derivative of the preference relation. Consider a cumulative distribution function $F_{1}$. Under suitable differentiability conditions (for the weak topology), one can approx-

${ }^{22}$ This divergence is clearly due to the nonlinear weighting functions since fanning out and strict fanning out are equivalent for linear indifference curves. 
imate the preferences for lotteries $F_{2}$ close to $F_{1}$ by a linear preference:

$$
V\left(F_{2}\right) \simeq V\left(F_{1}\right)+\int U_{F_{1}}(x)\left[d F_{2}(x)-d F_{1}(x)\right],
$$

where $U_{F_{1}}(\cdot)$ is a function that represents the derivative of $V$ at $F_{1}$.

This amounts to saying that in a neighborhood of a distribution $F_{1}$, preferences can be approximated by an expected utility model with utility function $U_{F_{1}}$, referred to as the local utility function at $F_{1}$.

Moreover, Machina's analysis shows that many properties of the expected utility model extend to nonlinear differentiable preferences when applied to the whole set of local utility functions $\left\{U_{F}(\cdot)\right\}_{F}$. For example, $V$ is risk-averse if all local utility functions are concave.

For a CPT model with utility function $u(x)$ and weighting functions $G$ and $H$, the local utility functions are given by

$$
U_{F}^{\prime}(x)=u^{\prime}(x) G^{\prime}(1-F(x)) \text { if } x>0
$$

and

$$
U_{F}^{\prime}(x)=u^{\prime}(x) H^{\prime}(F(x)) \text { if } x<0 .
$$

The most interesting feature of these utility functions is their (local) risk aversion. Assuming that $F$ has a well-defined density $f$ (which is, of course, not the case for horse races), we find that

$$
U_{F}^{\prime \prime}(x)=u^{\prime \prime}(x) G^{\prime}(1-F(x))-u^{\prime}(x) G^{\prime \prime}(1-F(x)) f(x)
$$

if $x>0$ and

$$
U_{F}^{\prime \prime}(x)=u^{\prime \prime}(x) H^{\prime}(F(x))+u^{\prime}(x) H^{\prime \prime}(F(x)) f(x)
$$

if $x<0$. Thus, as pointed out by Chew, Karni, and Safra (1987), local risk aversion depends on the concavity both of the utility function $u$ and of the weighting functions $G$ and $H$. More precisely, the local utility function $U_{F}$ will be risk-averse for gains if $u$ is concave and $G$ is convex and risk-averse for losses if $u$ is concave and $H$ is concave.

Now our estimated $u$ is convex, which suggests risk loving; but our estimated weighting functions point in the direction of risk aversion since $G$ is (slightly) convex and $H$ is (clearly) concave. This suggests that for some distributions, the local utility function will exhibit risk aversion, especially for losses, since $H$ is highly concave.

It is difficult to go further in our application since our distributions $F$ have discrete support. However, we can easily derive local utility functions for hypothetical continuous distributions. As a simple illustration, consider the uniform distribution on $[-B, B]$, which 
has a cumulative distribution function $F(x)=(x+B) / 2 B$. The formulas given above imply that

$$
U_{F}^{\prime}(x)=\exp (-\theta x) \alpha\left(\frac{B-x}{2 B}\right)^{\alpha-1}
$$

if $x>0$ and

$$
U_{F}^{\prime}(x)=\exp (-\theta x) \beta\left(\frac{x+B}{2 B}\right)^{\beta-1}
$$

if $x<0$. Differentiating them, we find that the sign of $V_{F}^{\prime \prime}(x)$ is given by the expressions

$$
-\theta-\frac{\alpha-1}{B-x}
$$

if $x>0$ and

$$
-\theta+\frac{\beta-1}{x+B}
$$

if $x<0$. Now recall that only $\theta^{\prime}=\theta a$ (where $a$ is the unknown sign of the bet) is identified in our estimating procedure, and define $\lambda$ $=B / a$. The parameter $\lambda$ measures how large the global risk on the individual's wealth is relative to a bet on horse races. We define two critical values for $\lambda$ :

$$
\begin{aligned}
& \lambda_{L}=-\frac{\alpha-1}{\theta^{\prime}} \simeq 2.26, \\
& \lambda_{H}=-\frac{1-\beta}{\theta^{\prime}} \simeq 9.51 .
\end{aligned}
$$

Given this notation, simple calculations show that $U_{F}^{\prime \prime}(x)$ has the sign of

$$
(B+x)-B \frac{\lambda_{H}}{\lambda}
$$

for losses and

$$
(B-x)-B \frac{\lambda_{L}}{\lambda}
$$

for gains. Thus there are only three possible cases: (1) if global risks are small $\left(\lambda<\lambda_{L}\right)$, the local utility function exhibits risk aversion on the whole interval $[-B, B]$; (2) for intermediate values $\left(\lambda_{L}<\lambda\right.$ $<\lambda_{H}$ ), it is risk-averse for losses and for large gains but risk-loving 


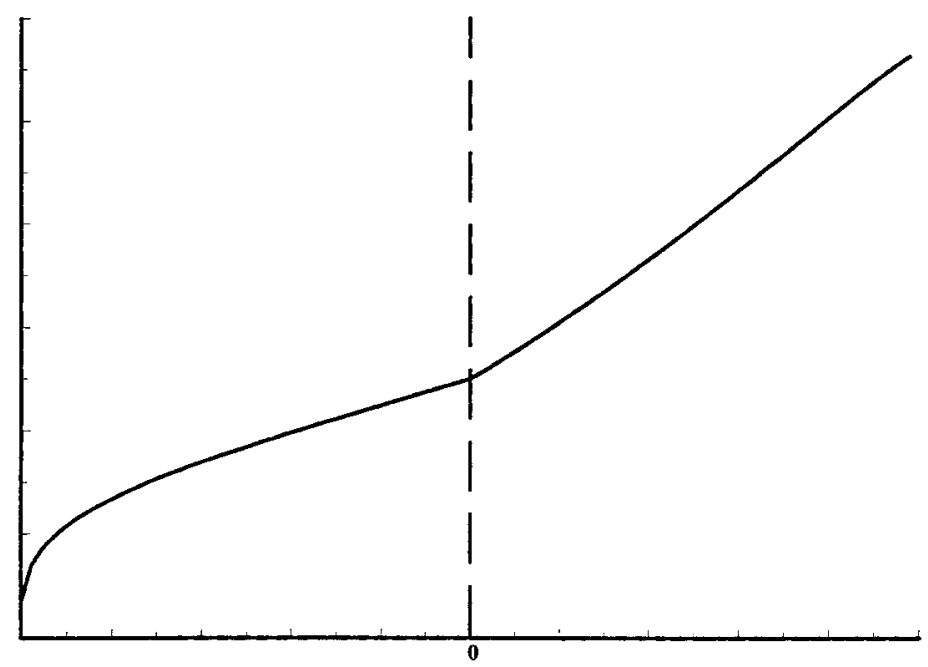

FIg. 8.- Shape of the local utility function

for small gains; and (3) when global risks are large $\left(\lambda>\lambda_{H}\right)$, it is risk-loving for small losses and gains and risk-averse for large losses and gains.

Although this particular exercise cannot pretend to realism, the last case seems to be the most interesting since global risks are bound to be much larger than the size of a bet on a horse. Our estimated local utility function, as illustrated in figure 8 for $\lambda=B=10$, then has a shape similar to those proposed by Friedman and Savage (1948) and Markowitz (1952) and later discussed by Machina (1982) in the context of non-expected utility models. ${ }^{23}$ In other words, they are consistent with the observation that individuals simultaneously insure some risk and gamble, at any level of wealth. Note in particular that, for all values of $\lambda$, agents appear to be risk-averse for large losses: this means that the agent would insure against a risk of a large loss that occurs with a small probability. One can obtain a similar graph (numerically) when $F$ is a centered normal distribution.

\section{What Features of the Data Do We Fit?}

Since our results are based on maximum likelihood estimation, we in effect fit the whole shape of the probabilities-odds equilibrium relationship given in (1). The favorite/long shot bias is perhaps the

\footnotetext{
${ }^{23}$ It is also reminiscent of the (expected utility) estimates of Golec and Tamarkin (1998).
} 


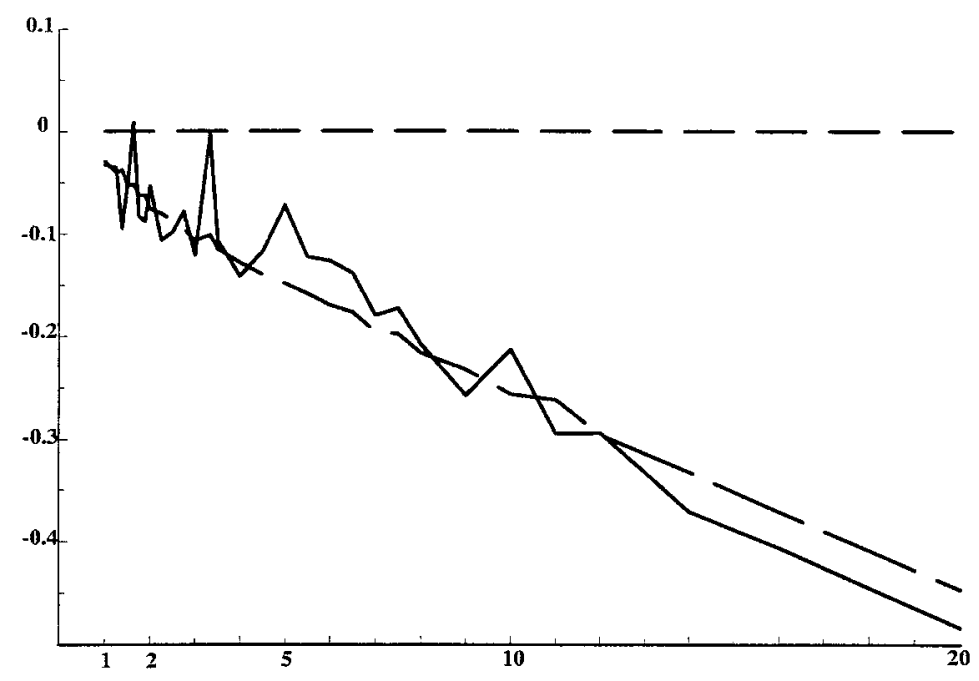

FIG. 9.-Replicating the favorite/long shot bias

best-known "anomaly" in horse race betting. Since our estimation procedure does not rely on it, it is interesting to see whether our estimates reproduce anything like the observed expected return curve in figure 1 . To check this, for all $R$ from 0.02 to 20 by steps of 0.01 , we compute the probabilities $p_{i}\left(\mathbf{R}^{C}, \hat{\boldsymbol{\theta}}\right)$ for all races $c$ in 1995 and horses $i$ such that $R_{i}^{C}=R$. We then estimate the probability of winning with rate of return $R$ by $\tilde{p}(R)$, the average of all such probabilities, and we estimate the expected return by

$$
\widetilde{\mathrm{ER}}(R)=\tilde{p}(R)(R+1)-1 .
$$

Figure 9 plots the result ${ }^{24}$ along with the $\overline{\mathrm{ER}}(R)$ curve from figure 1 .

Clearly, our CPT power estimates provide a very good fit for the observed expected return curve. Indeed, $\widetilde{\mathrm{ER}}(R)$ lies almost everywhere within the 95 percent confidence interval for $\overline{\mathrm{ER}}(R)$. It should be noted, however, that our CARA expected utility estimates provide about as good a visual fit. To go further, we generated for all horses that ran in 1995 (there are 40,118 of them) the best-fitting expected utility (respectively CPT) estimate of their probability of winning; we denote them $p_{\mathrm{EU}}$ and $p_{\mathrm{CPT}}$. We then ran a nonparametric regression of $p_{\mathrm{EU}}-p_{\mathrm{CPT}}$ on $p_{\mathrm{CPT}}$. The result ${ }^{25}$ is given in figure 10 . It appears that relative to CPT, expected utility overestimates the outsiders'

\footnotetext{
${ }^{24}$ For all rates of return that correspond to at least 100 horses in 1995.

${ }^{25}$ We dropped the horses for which $p_{\mathrm{CPT}}>0.5$, which represent less than 2 percent of the data.
} 


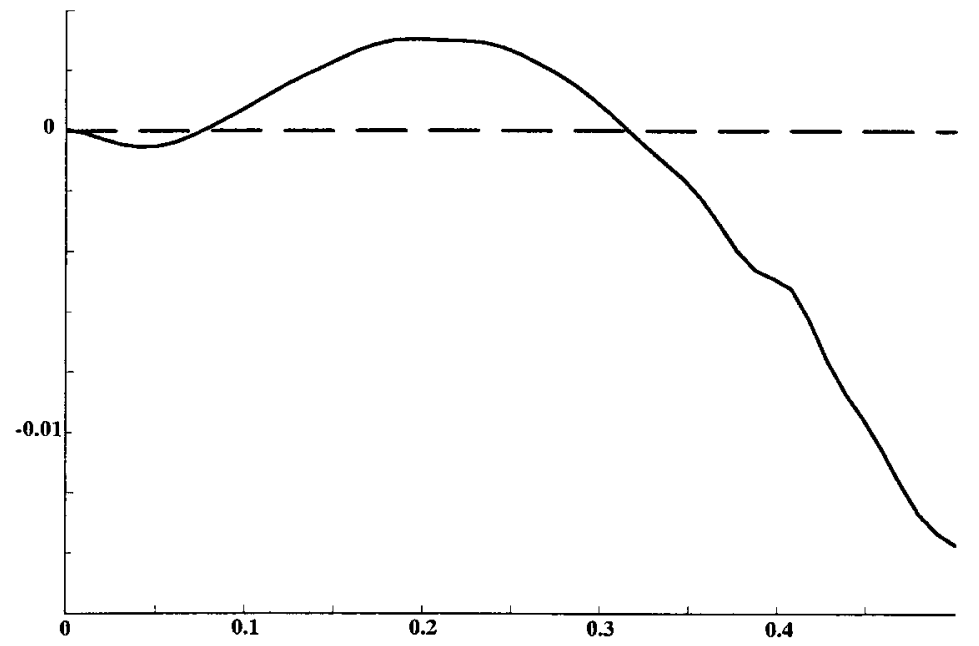

FIG. 10.-Nonparametric regression of $p_{\mathrm{EU}}-p_{\mathrm{CPT}}$ on $p_{\mathrm{CPT}}$

probability of winning and (much more strongly) underestimates the favorites' probability of winning. In that sense, our CPT estimates provide a better fit for the favorite/long shot bias than expected utility.

As a matter of fact, there are anomalies in the data that cannot be rationalized by any expected utility specification, contrary to the favorite/long shot bias. Indeed, note that for expected utility, (1) implies, for any two horses $i$ and $j$ in the same race,

$$
\frac{p_{i}}{p_{j}}=\frac{u\left(M+a R_{j}\right)-u(M-a)}{u\left(M+a R_{i}\right)-u(M-a)}
$$

so that the relative probability of winning depends only on $R_{i}$ and $R_{j}$, and not on other characteristics of the race. Therefore, one simple nonparametric test of expected utility would be to choose two odds, $R$ and $R^{\prime}$; to select all races in which there are two horses with respective odds $R$ and $R^{\prime}$; to compute the number of horses $N\left(N^{\prime}\right)$ with odds $R\left(R^{\prime}\right)$ in these races that won; and to check whether $N / N^{\prime}$ depends on, say, the number of horses in the race. It is easy to find such violations of expected utility in the data. Our CPT estimates presumably also are driven by these "anomalies."

\section{Concluding Remarks}

We have estimated only a few specifications here. The very high computation costs involved in estimating the CPT model make it difficult 
to explore it much further. On the other hand, while the logical progression from expected utility to RDEU (which nests expected utility) and then to CPT (which nests RDEU) is intellectually satisfying, there exist other classes of models. Weighted expected utility is a possibility since it leads to much simpler computations than CPT. However, weighted expected utility implies a "generalized linearity", property that contradicts our CPT estimates. This is why we did not estimate it here. Note also that nonadditive models of uncertainty aversion, as in Schmeidler (1989), cannot be estimated using our procedure since our setup involves risky and not uncertain choices.

Clearly, the greatest weakness of our paper is the representative agent assumption, even though we imposed it only for a subgroup of the population of bettors. However, relaxing this assumption would require detailed information on which bettor, with what characteristics, bet on which horse in which race. It would also raise difficult econometric problems since it may not be easy to exploit information that reduces to "bettor $i$ bet on horse $j$ in race $c$."

\section{Appendix}

We prove here that the set of equalities (1) defines probabilities uniquely as a function of odds if the functional $V$ is continuous in $F$ and exhibits first-order stochastic dominance. To do this, we solve the system in two steps. We first introduce an auxiliary unknown $w$ such that, for all $i=1$, $\ldots, n, W\left(p_{i}, R_{i}, \boldsymbol{\theta}\right)=w$. We shall prove that this determines functions $p_{i}$ $=P\left(w, R_{i}, \boldsymbol{\theta}\right)$. We then use the constraint $\sum_{i=1}^{n} P\left(w, R_{i}, \boldsymbol{\theta}\right)$ to determine the remaining unknown $w$ as a function of $\mathbf{R}$ and $\boldsymbol{\theta}$.

First note that, given first-order stochastic dominance and continuity, $W\left(p_{i}, R_{i}, \boldsymbol{\theta}\right)$ is a continuous increasing function of its first argument. Therefore, $P\left(w, R_{i}, \boldsymbol{\theta}\right)$ is a well-defined function for $w$ in the interval $\left[W\left(0, R_{i}\right.\right.$, $\left.\boldsymbol{\theta}), W\left(1, R_{i}, \boldsymbol{\theta}\right)\right]$, and it is continuous and increasing in $w$. It follows that the solution $w$ to $\sum_{i=1}^{n} P\left(w, R_{i}, \boldsymbol{\theta}\right)=1$ must be unique if it exists.

Now consider $w$ and $i$ such that $w=W\left(1, R_{i}, \boldsymbol{\theta}\right)$. Clearly, this implies $P\left(w, R_{i}, \boldsymbol{\theta}\right)=1$ and, therefore, $\sum_{j=1}^{n} P\left(w, R_{j}, \boldsymbol{\theta}\right)>1$. Now note that all $W\left(0, R_{i}, \boldsymbol{\theta}\right)$ are equal since they represent the value of losing $£ a$ with probability one. Therefore, if

$$
w=W\left(0, R_{1}, \boldsymbol{\theta}\right)=W\left(0, R_{n}, \boldsymbol{\theta}\right),
$$

we must have $P\left(w, R_{i}, \boldsymbol{\theta}\right)=0$ for all $i$ and thus $\sum_{i=1}^{n} P\left(w, R_{i}, \boldsymbol{\theta}\right)<1$. This concludes the proof by showing that there exists a unique $w(\mathbf{R}, \boldsymbol{\theta})$ in the interval $\left(W\left(0, R_{i}, \boldsymbol{\theta}\right), \min _{i=1, \ldots, n} W\left(1, R_{i}, \boldsymbol{\theta}\right)\right)$ that solves the system of equalities. It is then a simple task to obtain the probabilities

$$
p_{i}(\mathbf{R}, \boldsymbol{\theta})=P\left(w(\mathbf{R}, \boldsymbol{\theta}), R_{i}, \boldsymbol{\theta}\right) .
$$

Q.E.D. 


\section{References}

Ali, Mukhtar M. "Probability and Utility Estimates for Racetrack Bettors." J.P.E. 85 (August 1977): 803-15.

$\rightarrow$ _ . "Some Evidence of the Efficiency of a Speculative Market." Econometrica 47 (March 1979): 387-92.

Allais, Maurice. "Le comportement de l'homme rationnel devant le risque: critique des postulats et axiomes de l'école américaine." Econometrica 21 (October 1953): 503-46. English translation in The Expected Utility Hypotheses and the Allais Paradox: Contemporary Discussions of Decisions under Uncertainty with Allais' Rejoinder, edited by Maurice Allais and Ole Hagen. Boston: Reidel, 1979.

Camerer, Colin F. "Individual Decision Making." In The Handbook of Experimental Economics, edited by John H. Kagel and Alvin E. Roth. Princeton, N.J.: Princeton Univ. Press, 1995.

$\rightarrow$ Camerer, Colin F., and Ho, Teck-Hua. "Violations of the Betweenness Axiom and Nonlinearity in Probability." J. Risk and Uncertainty 8 (March 1994): 167-96.

$\rightarrow$ Carbone, Enrica, and Hey, John D. "A Comparison of the Estimates of Expected Utility and Non-Expected-Utility Preference Functionals." Geneva Papers Risk and Insurance Theory 20, no. 1 (1995): 111-33.

$\rightarrow$ Chew, Soo Hong; Karni, Edi; and Safra, Zvi. "Risk Aversion in the Theory of Expected Utility with Rank Dependent Probabilities." J. Econ. Theory 42 (August 1987): 370-81.

$\rightarrow$ Cicchetti, Charles J., and Dubin, Jeffrey A. "A Microeconometric Analysis of Risk Aversion and the Decision to Self-Insure." J.P.E. 102 (February 1994): 169-86.

$\rightarrow$ Crafts, N. F. R. "Some Evidence of Insider Knowledge in Horse Race Betting in Britain." Economica 52 (August 1985): 295-304.

$\rightarrow$ Dowie, Jack A. "On the Efficiency and Equity of Betting Markets." Economica 43 (May 1976): 139-50.

$\rightarrow$ Epstein, Larry G., and Zin, Stanley E. "Substitution, Risk Aversion, and the Temporal Behavior of Consumption and Asset Returns: An Empirical Analysis." J.P.E. 99 (April 1991): 263-86.

Fennema, Hein, and Wakker, Peter P. "A Test of Rank-Dependent Utility in the Context of Ambiguity." J. Risk and Uncertainty 13 (July 1996): 1935.

$\rightarrow$ Friedman, Milton, and Savage, Leonard J. "The Utility Analysis of Choices Involving Risk.” J.P.E. 56 (August 1948): 279-304.

$\rightarrow$ Golec, Joseph, and Tamarkin, Maurry. "Bettors Love Skewness, Not Risk, at the Horse Track." J.P.E. 106 (February 1998): 205-25.

Gollier, Christian, and Machina, Mark J., eds. Non-Expected Utility and Risk Management: A Special Issue of the Geneva Papers on Risk and Insurance. Boston: Kluwer, 1995.

$\rightarrow$ Green, Jerry R., and Jullien, Bruno. "Ordinal Independence in Nonlinear Utility Theory." J. Risk and Uncertainty 1 (December 1988): 355-87. [“Erratum." J. Risk and Uncertainty 2 (April 1989): 119.]

$\rightarrow$ Griffith, R. M. "Odds Adjustments by American Horse-Race Bettors." American J. Psychology 62 (April 1949): 290-94.

Hausch, Donald B.; Lo, Victor S. Y.; and Ziemba, William T. Efficiency of Racetrack Betting Markets. London: Academic Press, 1994.

$\rightarrow$ Hey, John D., and Orme, Chris. "Investigating Generalizations of Expected 
Utility Using Experimental Data." Econometrica 62 (November 1994): 1291-1326.

Jorion, Philippe, and Giovannini, Alberto. "Time-Series Tests of a NonExpected-Utility Model of Asset Pricing.” European Econ. Rev. 37 (June 1993): 1083-1100.

$\rightarrow$ Jullien, Bruno, and Salanié, Bernard. "Measuring the Incidence of Insider Trading: A Comment on Shin.” Econ. J. 104 (November 1994): 1418-19.

$\rightarrow$ Kahneman, Daniel, and Tversky, Amos. "Prospect Theory: An Analysis of Decision under Risk." Econometrica 47 (March 1979): 263-91.

$\rightarrow$ Lattimore, Pamela K.; Baker, Joanna R.; and Witte, Ann D. "The Influence of Probability on Risky Choice: A Parametric Examination." J. Econ. Behavior and Organization 17 (May 1992): 377-400.

$\rightarrow$ Machina, Mark J. " 'Expected Utility' Analysis without the Independence Axiom." Econometrica 50 (March 1982): 277-323.

$\rightarrow$ _ . "Choice under Uncertainty: Problems Solved and Unsolved." J. Econ. Perspectives 1 (Summer 1987): 121-54.

$\rightarrow$ - "Dynamic Consistency and Non-Expected Utility Models of Choice under Uncertainty." J. Econ. Literature 27 (December 1989): 1622-68.

$\rightarrow$ Markowitz, Harry M. "The Utility of Wealth." J.P.E. 60 (April 1952): 15158.

Marschak, Jacob. "Rational Behavior, Uncertain Prospects, and Measurable Utility." Econometrica 18 (April 1950): 111-41. ["Errata."' Econometrica 18 (July 1950): 312.]

$\rightarrow$ Prelec, Drazen. "The Probability Weighting Function." Econometrica 66 (May 1998): 497-527.

$\rightarrow$ Quiggin, John. "A Theory of Anticipated Utility." J. Econ. Behavior and Organization 3 (December 1982): 323-43.

$\rightarrow$ Schmeidler, David. "Subjective Probability and Expected Utility without Additivity." Econometrica 57 (May 1989): 571-87.

$\rightarrow$ Segal, Uzi. "Some Remarks on Quiggin's Anticipated Utility." J. Econ. Behavior and Organization 8 (March 1987): 145-54.

$\rightarrow$ Shin, Hyun Song. "Measuring the Incidence of Insider Trading in a Market for State-Contingent Claims.”' Econ. J. 103 (September 1993): 1141-53.

$\rightarrow$ Tversky, Amos, and Kahneman, Daniel. "Advances in Prospect Theory: Cumulative Representation of Uncertainty." J. Risk and Uncertainty 5 (October 1992): 297-323.

$\rightarrow$ Wakker, Peter P., and Tversky, Amos. "An Axiomatization of Cumulative Prospect Theory." J. Risk and Uncertainty 7 (October 1993): 147-75.

$\rightarrow$ Weil, Philippe. "Nonexpected Utility in Macroeconomics." Q.J.E. 105 (February 1990): 29-42.

$\rightarrow$ Weitzman, Martin. "Utility Analysis and Group Behavior: An Empirical Study." J.P.E. 73 (February 1965): 18-26.

Wu, George. "An Empirical Test of Ordinal Independence." J. Risk and Uncertainty 9 (July 1994): 39-60.

Yaari, Menahem E. "The Dual Theory of Choice under Risk.' Econometrica 55 (January 1987): 95-115. 